This item was submitted to Loughborough's Research Repository by the author.

Items in Figshare are protected by copyright, with all rights reserved, unless otherwise indicated.

\title{
A snapping cosmic string in a de Sitter or anti-de Sitter universe
}

PLEASE CITE THE PUBLISHED VERSION

LICENCE

CC BY-NC-ND 4.0

\section{REPOSITORY RECORD}

Podolsky, J., and J.B. Griffiths. 2019. "A Snapping Cosmic String in a De Sitter or Anti-de Sitter Universe". figshare. https://hdl.handle.net/2134/237. 


\title{
A snapping cosmic string in a de Sitter or anti-de Sitter universe
}

\author{
J. Podolský* \\ Institute of Theoretical Physics, Charles University in Prague, \\ V Holešovičkách 2, 18000 Prague 8, Czech Republic. \\ and J. B. Griffiths ${ }^{\dagger}$ \\ Department of Mathematical Sciences, Loughborough University \\ Loughborough, Leics. LE11 3TU, U.K.
}

March 19, 2004

\begin{abstract}
We present and describe an exact solution of Einstein's equations which represents a snapping cosmic string in a vacuum background with a cosmological constant $\Lambda$. The snapping of the string generates an impulsive spherical gravitational wave which is a particular member of a known family of such waves. The global solution for all values of $\Lambda$ is presented in various metric forms and interpreted geometrically. It is shown to represent the limit of a family of sandwich type $\mathrm{N}$ Robinson-Trautman waves. It is also derived as a limit of the C-metric with $\Lambda$, in which the acceleration of the pair of black holes becomes unbounded while their masses are scaled to zero.
\end{abstract}

\section{Introduction}

An exact solution of Einstein's equations is known [1], [2] which describes an impulsive spherical gravitational wave in a Minkowski background that is generated by a snapping infinite cosmic string (or by an expanding string inside the sphere [3]). This solution can be obtained either [1] by pasting two appropriate forms of Minkowski space either side of the spherical wavefront, or [2] as a limiting case of particular solutions with boost-rotation symmetry (see also [3]-[5]). In both constructions, the two ends of the semi-infinite strings recede from a common point generating an impulsive spherical wave. (Alternatively, there is an expanding cosmic string along the axis of symmetry separating the two particles.) However, as already pointed out [2], this solution does not strictly describe a snapping cosmic string, but rather two semi-infinite cosmic strings which initially approach at the speed of light and then separate again at the instant at which they collide. The purpose of the present paper is to present and investigate an analogous solution which describes a snapping cosmic string in a de Sitter or anti-de Sitter universe, thus generating an impulsive spherical wave in either of these backgrounds.

In fact, a general method for constructing expanding impulsive spherical gravitational waves in a Minkowski background had previously been suggested by Penrose [6]. This involves cutting Minkowski space-time along a null cone and then re-attaching the two pieces with a suitable "warp". The explicit general solution written in a continuous coordinate system is given in [7]-[10]. This, of course, includes the solution which describes a snapping cosmic string as a particular case.

${ }^{*}$ E-mail: Podolsky@mbox.troja.mff.cuni.cz

${ }^{\dagger}$ E-mail: J.B.Griffiths@Lboro.ac.uk 
This general family of solutions has also been extended to include a non-zero cosmological constant $\Lambda$ [11], [12]. These solutions describe a family of expanding spherical gravitational waves in a de Sitter or anti-de Sitter universe. In this paper, we identify the specific solution which represents a snapping cosmic string in these backgrounds. Before presenting this, however, we first describe an instructive geometrical representation of a cosmic string in the (anti-)de Sitter background.

Further, the general class of solutions of this type has been shown [13] to be equivalent to impulsive limits of the class of Robinson-Trautman type $\mathrm{N}$ solutions. In section 4, we will present the exact solution for a snapping string in this context. In section 5 , we will demonstrate how this solution can also be obtained as a limit of the C-metric which can be interpreted as the field of two black holes accelerating away from each other under the action of strings which pull in opposite directions. In the limit as the acceleration $A$ becomes unbounded while the mass $m$ of the black holes is reduced to zero keeping the quantity $m A$ constant, this reduces exactly to the solution for a snapping cosmic string. For a Minkowski background, this procedure is known [5]. However, the method applied there cannot be extended to the case when the cosmological constant is non-zero as no global coordinate system (analogous to that for the Weyl coordinates) is known for this case. A different method is therefore developed in section 5 in which the solution for a snapping cosmic string in a de Sitter or anti-de Sitter background can be derived as the limit of the generalization of the C-metric which contains a cosmological constant.

\section{A 5-dimensional representation}

As is well known, the de Sitter and anti-de Sitter space-times can be represented as the 4dimensional hyperboloid

$$
Z_{0}^{2}-Z_{1}^{2}-Z_{23}^{2}-\varepsilon Z_{4}^{2}=-\varepsilon a^{2},
$$

in the flat 5-dimensional space

$$
\mathrm{d} s^{2}=\mathrm{d} Z_{0}^{2}-\mathrm{d} Z_{1}^{2}-\mathrm{d} Z_{23}{ }^{2}-Z_{23}{ }^{2} e^{-2 c} \mathrm{~d} \Phi^{2}-\varepsilon \mathrm{d} Z_{4}^{2},
$$

where $a^{2}=3 /|\Lambda|, \varepsilon$ is the sign of $\Lambda, c$ is a constant, and $Z_{23} \in[0, \infty)$ and $\Phi \in[0,2 \pi)$ are related to standard cartesian coordinates by $Z_{2}=Z_{23} \cos \Phi$ and $Z_{3}=Z_{23} \sin \Phi$. When $c=0$ and $\Lambda>0$, this is a complete de Sitter space in which, at any time $Z_{0}=$ const., the universe is closed and has the geometry of a 3 -sphere. When $c>0$, a wedge in the space-time is missing, and the de Sitter space may be considered to include a string with a deficit angle $2 \pi\left(1-e^{-c}\right)$ located along the line $Z_{23}=0$. It may immediately be observed from (1) that the string lies on a closed meridian around the universe. As the universe contracts (for $Z_{0}<0$ ) and then expands (for $Z_{0}>0$ ), the length of the string also correspondingly contracts and expands.

Analogously, when $c=0$ and $\Lambda<0$, this is the complete open anti-de Sitter space. When $c>0$, a wedge in the space-time is missing, and the space may be considered to include an infinite string with a deficit angle $2 \pi\left(1-e^{-c}\right)$ located along the line $Z_{23}=0$.

It will be shown in the following sections that the ends of a snapped cosmic string in both the de Sitter and the anti-de Sitter universe propagate along the null generators of the hyperboloid which are located on the section $Z_{4}=a$ which corresponds to the poles of an expanding sphere on which $Z_{1}^{2}+Z_{23}^{2}=Z_{0}^{2}$. This is illustrated in figure 1. In the de Sitter case, the string is contained in the region for which $Z_{4}<a$, but is absent for $Z_{4}>a$. However, for $\Lambda<0$, the string is contained in the region for which $Z_{4}>a$, and is absent for $Z_{4}<a$. For $Z_{4} \neq a$, such a situation can be formally represented by the line element

$$
\mathrm{d} s_{0}{ }^{2}=\mathrm{d} Z_{0}{ }^{2}-\mathrm{d} Z_{1}{ }^{2}-\mathrm{d} Z_{23}{ }^{2}-Z_{23}{ }^{2} e^{-2 c \Theta\left(\varepsilon\left(a-Z_{4}\right)\right)} \mathrm{d} \Phi^{2}-\varepsilon \mathrm{d} Z_{4}{ }^{2},
$$

where $\Theta$ is the Heaviside step function. However, the metric (3) does not correspond to a global solution of Einstein's equations. The discontinuity across $Z_{4}=a$ must be accompanied by 

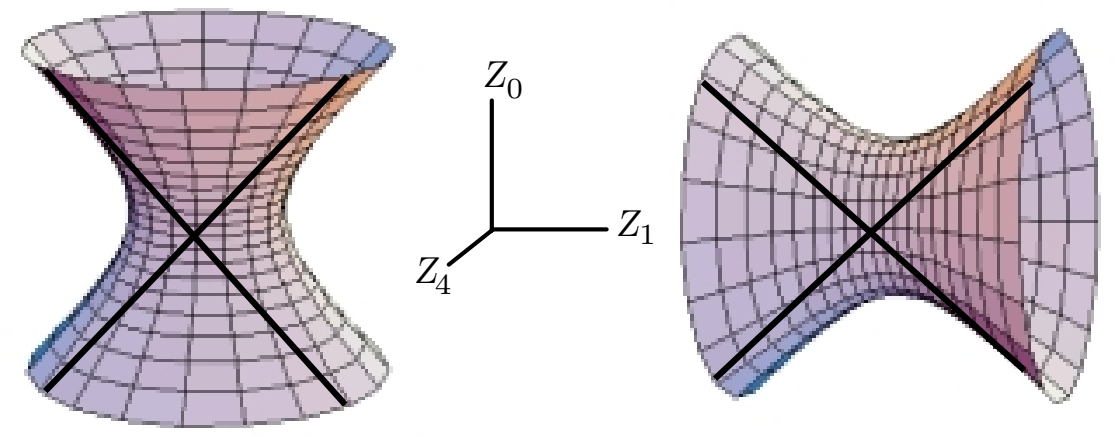

Figure 1: The hyperboloids represent the de Sitter and anti-de Sitter backgrounds with $Z_{23}=0$. The expanding spherical impulsive waves are located on the sections with $Z_{4}=a$. In these sections, strings are located in the region with $Z_{4}<a$ in the de Sitter background and $Z_{4}>a$ in the anti-de Sitter background.

an impulsive component which can be interpreted as an expanding spherical gravitational wave. The complete exact solution will be presented below.

\section{An explicit solution in a continuous metric form}

We will now use the Penrose "cut and paste" method [6] (see also [7], [12] and [13]) to construct an explicit solution representing a snapping cosmic string in a background with a non-zero cosmological constant. It is convenient to represent the (anti-)de Sitter background by a line element in the form

$$
\mathrm{d} s^{2}=\frac{2 \mathrm{~d} \mathcal{U} \mathrm{d} \mathcal{V}-2 \mathrm{~d} \eta \mathrm{d} \bar{\eta}}{\left[1-\frac{1}{6} \Lambda(\mathcal{U} \mathcal{V}-\eta \bar{\eta})\right]^{2}}
$$

which is related to the parameterization of the hyperboloid (1) by the substitution

$$
\mathcal{U}=\sqrt{2} a \frac{Z_{0}+Z_{1}}{Z_{4}+a}, \quad \mathcal{V}=\sqrt{2} a \frac{Z_{0}-Z_{1}}{Z_{4}+a}, \quad \eta=\sqrt{2} a \frac{Z_{23} \exp (i \Phi)}{Z_{4}+a} .
$$

Starting with the line element (4), we first perform the transformation

$$
\mathcal{U}=U+V Z \bar{Z} / p, \quad \mathcal{V}=V / p+\epsilon U, \quad \eta=V Z / p,
$$

where

$$
p=1+\epsilon Z \bar{Z}, \quad \epsilon=-1,0,1 .
$$

(It can be seen from [16] that the most natural choice of parameter for these solutions is $\epsilon=1$. However, in the impulsive limit, all choices are permitted and the parameter $\epsilon$ is retained below.) With the transformation (5), (4) becomes

$$
\mathrm{d} s^{2}=\frac{2 \mathrm{~d} U \mathrm{~d} V+2 \epsilon \mathrm{d} U^{2}-2(V / p)^{2} \mathrm{~d} Z \mathrm{~d} \bar{Z}}{\left[1-\frac{1}{6} \Lambda U(V+\epsilon U)\right]^{2}} .
$$

The alternative transformation

$$
\begin{aligned}
\mathcal{V} & =\frac{|Z|^{\delta-2}}{1-\delta}\left(\frac{|Z|^{2}}{p} V+\left[\left(\frac{\delta}{2}\right)^{2}+\left(1-\frac{\delta}{2}\right)^{2} \epsilon|Z|^{2}\right] U\right), \\
\mathcal{U} & =\frac{|Z|^{-\delta}}{1-\delta}\left(\frac{|Z|^{2}}{p} V+\left[\left(1-\frac{\delta}{2}\right)^{2}+\left(\frac{\delta}{2}\right)^{2} \epsilon|Z|^{2}\right] U\right), \\
\eta & =\frac{Z^{1-\delta}|Z|^{\delta-2}}{1-\delta}\left(\frac{|Z|^{2}}{p} V+\left(\frac{\delta}{2}\right)\left(1-\frac{\delta}{2}\right) p U\right),
\end{aligned}
$$


where $\delta \in[0,1)$ is a constant parameter, puts the metric (4) into the form

$$
\mathrm{d} s^{2}=\frac{2 \mathrm{~d} U \mathrm{~d} V+2 \epsilon \mathrm{d} U^{2}-2|(V / p) \mathrm{d} Z-U p \bar{H} \mathrm{~d} \bar{Z}|^{2}}{\left[1-\frac{1}{6} \Lambda U(V+\epsilon U)\right]^{2}},
$$

where

$$
H=\frac{\frac{\delta}{2}\left(1-\frac{\delta}{2}\right)}{Z^{2}}
$$

Apart from some trivial changes of notation, this corresponds to the general transformation given in [12] with the specific choice $h(Z)=Z^{1-\delta}$ which, when $\delta \neq 0$, introduces a cosmic string with deficit angle $2 \pi \delta$.

We now combine the above line elements by attaching (6) for $U>0$ with (8) for $U<0$. The resulting metric is expressed explicitly as

$$
\mathrm{d} s^{2}=\frac{2 \mathrm{~d} U \mathrm{~d} V+2 \epsilon \mathrm{d} U^{2}-2|(V / p) \mathrm{d} Z-U \Theta(-U) p \bar{H} \mathrm{~d} \bar{Z}|^{2}}{\left[1-\frac{1}{6} \Lambda U(V+\epsilon U)\right]^{2}} .
$$

The line element (10) is continuous in $U$. However, there is a discontinuity in the derivatives of the metric on the null hypersurface $U=0$. This yields an impulsive component in the curvature tensor component $\Psi_{4}=\left(p^{2} H / V\right) \delta(U)$ using a natural tetrad, indicating the presence of an impulsive gravitational wave on this hypersurface. In the above metrics, this hypersurface represents an expanding sphere given by $\mathcal{U} \mathcal{V}-\eta \bar{\eta}=0$. In the de Sitter or anti-de Sitter background, this corresponds to the section $Z_{4}=a$ in accordance with the geometrical description given in section 2 (see figure 1). Moreover, since

$$
U(V+\epsilon U)=\mathcal{U V}-\eta \bar{\eta}=\frac{6}{\Lambda} \frac{\left(Z_{4}-a\right)}{\left(Z_{4}+a\right)},
$$

for $V+\epsilon U>0$, it can be seen that the region inside the spherical wavefront, for which $U>0$, corresponds to $Z_{4}>a$ in a de Sitter background, but to $Z_{4}<a$ in an anti-de Sitter background. Similarly, the outside region $U<0$ which contains the strings corresponds to $Z_{4}<a$ when $\Lambda>0$ and to $Z_{4}>a$ when $\Lambda<0$.

In the combined metric (10), the argument of the complex coordinate $Z$ has the full range $\arg Z \in[0,2 \pi)$ everywhere. In the region inside the spherical wave in which $U>0$, it can be seen from (5) that $\Phi=\arg \eta=\arg Z$ so that, for this region, $\Phi$ has the same full range $[0,2 \pi)$. Thus, inside the spherical wave, the space-time is locally complete, corresponding to $c=0$ in (2). However, in the outer region where $U<0$, the expression for $\eta$ in (7) implies that $\Phi=\arg \eta=(1-\delta) \arg Z$ so that here $\Phi \in[0,(1-\delta) 2 \pi)$. Thus, for $\delta \in(0,1)$, a wedge of the space-time is missing. In order to express this in the form (2), it is necessary to rescale the coordinate $\Phi$. This is equivalent to setting a non-zero value for $c$ such that $e^{-c}=1-\delta$, corresponding to the presence of a string with deficit angle $2 \pi \delta$ in the region $U<0$ outside the impulsive wave.

\section{Limit of Robinson-Trautman sandwich waves}

It was argued in [13] that the above class of solutions for expanding impulsive spherical gravitational waves can be considered to be impulsive limits of the family of vacuum RobinsonTrautman type N solutions with a cosmological constant. This has been further clarified in [15] and [16] using the standard form of the line element

$$
\mathrm{d} s^{2}=2 \mathrm{~d} u \mathrm{~d} r+\left[2 \epsilon-2 r(\log P)_{u}-\frac{\Lambda}{3} r^{2}\right] \mathrm{d} u^{2}-2 \frac{r^{2}}{P^{2}} \mathrm{~d} \zeta \mathrm{d} \bar{\zeta}
$$

where the function $P(\zeta, \bar{\zeta}, u)$ is given by

$$
P=(1+\epsilon F \bar{F})\left(F_{\zeta} \bar{F}_{\bar{\zeta}}\right)^{-1 / 2},
$$


in which $F=F(\zeta, u)$ is an arbitrary complex function of $u$ and $\zeta$, holomorphic in $\zeta$. In [16], we considered the particularly simple case in which

$$
F(\zeta, u)=\zeta^{g(u)}
$$

where $g(u)$ is an arbitrary positive function of retarded time. For this choice, we obtain

$$
P^{2}=\frac{\left[1+\epsilon(\zeta \bar{\zeta})^{g}\right]^{2}}{g^{2}(\zeta \bar{\zeta})^{g-1}}, \quad(\log P)_{u}=-\frac{g^{\prime}}{g}\left(1+\frac{1}{2} \log (\zeta \bar{\zeta})^{g}\left[\frac{1-\epsilon(\zeta \bar{\zeta})^{g}}{1+\epsilon(\zeta \bar{\zeta})^{g}}\right]\right)
$$

so that the metric is obviously axially symmetric, and the only non-zero component of the Weyl tensor using a natural tetrad is given by

$$
\bar{\Psi}_{4}=-\frac{1}{2 r} \frac{\bar{\zeta}}{\zeta}\left(\frac{1}{|\zeta|^{g}}+\epsilon|\zeta|^{g}\right)^{2} \frac{g^{\prime}}{g} .
$$

For regions in which $g$ is a constant, this solution is conformally flat and describes part of a Minkowski, de Sitter or anti-de Sitter background. For a more general $g(u)$ it represents an exact gravitational wave with amplitude given by (14). On any wave surface $u=$ const., the complex number $F=\zeta^{g}$ represents a stereographic-type coordinate. If we assume that the argument of $\zeta$ covers the full range $[0,2 \pi)$, then $\arg F \in[0,2 \pi g)$ and these surfaces include a deficit angle $2 \pi(1-g)$ about the symmetry axis $F=0$.

It is therefore a simple matter to construct the above model for a snapping cosmic string by setting $g=1$ for $u>0$, and $g=e^{-c}$ for $u<0$. This can be expressed as

$$
g(u)=e^{-c \Theta(-u)},
$$

so that $g^{\prime} / g=c \delta(u)$ and the Weyl tensor (14) only has an impulsive component on $u=0$. Although this produces a discontinuity in the metric function $P$ and an impulsive component in $(\log P)_{u}$, this construction may clearly be interpreted as the limit of a "well behaved" sandwich wave of the Robinson-Trautman family in which the metric is continuous.

The geometrical structure of these solutions and the character of the singularities for different values of $\epsilon$ and $\Lambda$ have been described in [16] for any $g(u)$. Here, we apply this for an impulsive spherical wave generated by a snapping string in a de Sitter or anti-de Sitter background.

It is known that the metric (11) can also be expressed in terms of García-Plebański coordinates [14], in the form

$$
\begin{gathered}
\mathrm{d} s^{2}=2 \\
+r\left[\left(f_{\xi}+\bar{f}_{\bar{\xi}}\right)-\frac{2 \epsilon}{\psi}(\bar{\xi} f+\xi \bar{f})\right] \mathrm{d} u^{2}
\end{gathered}
$$

where $f(\xi, u)$ is an arbitrary holomorphic function and $\psi=1+\epsilon \xi \bar{\xi}$. This is achieved using the transformation $\xi=F(\zeta, u)$ such that $F_{u}=f(F(\zeta, u), u)$. With the above specific choice (12), this corresponds to

$$
f(\xi, u)=\frac{g^{\prime}}{g} \xi \log \xi
$$

(Notice that this form can be found in the list [17] of Robinson-Trautman solutions with symmetries corresponding to an axial symmetry.) In particular, with (15), which represents a snapping cosmic string accompanied by an impulsive spherical gravitational wave, the line element becomes

$$
\begin{array}{rl}
\mathrm{d} s^{2}=2 \mathrm{~d} u & \mathrm{~d} r+\left(2 \epsilon-\frac{\Lambda}{3} r^{2}\right) \mathrm{d} u^{2}-2 \frac{r^{2}}{(1+\epsilon \xi \bar{\xi})^{2}}|\mathrm{~d} \xi-c \xi \log \xi \delta(u) \mathrm{d} u|^{2} \\
+ & 2 c r\left[1+\frac{1-\epsilon \xi \bar{\xi}}{1+\epsilon \xi \bar{\xi}} \log |\xi|\right] \delta(u) \mathrm{d} u^{2} .
\end{array}
$$

Unfortunately, this form is mathematically problematic as it explicitly contains a square of a delta function corresponding to the impulsive gravitational wave localised on $u=0$. 


\section{$5 \quad$ Null limit of the C-metric}

Let us now consider the well known C-metric which describes two black holes which accelerate away from each other under the action of two semi-infinite strings which pull them from infinity. It has been shown [5] that the null limit of this solution (as the acceleration $A$ tends to infinity while the mass $m$ tends to zero) is the solution for a snapping cosmic string in a Minkowski background. The generalisation of the C-metric to include a cosmological constant is well known. It is therefore appropriate to investigate whether a solution for a snapping cosmic string in a de Sitter or anti-de Sitter background can similarly be constructed by taking the null limit of this solution. However, it is found that the methods used in a Minkowski background do not extend to the case in which the cosmological constant is non-zero. For this case, a more complicated procedure is required.

The C-metric with a cosmological constant can be expressed by the line element

$$
\mathrm{d} s^{2}=\frac{1}{A^{2}(x+y)^{2}}\left(F \mathrm{~d} t^{2}-\frac{1}{F} \mathrm{~d} y^{2}-G \mathrm{~d} \phi^{2}-\frac{1}{G} \mathrm{~d} x^{2}\right),
$$

where

$$
G(x)=1-x^{2}-2 m A x^{3}, \quad F(y)=-\frac{\Lambda}{3 A^{2}}-1+y^{2}-2 m A y^{3} .
$$

This is included in the general class of solutions given in [18]. A physical interpretation and analysis of these space-times has been presented very recently in [19]-[23] where references to previous work can be found.

In the above form of the metric, however, it is not possible to retain the cosmological constant in the limit as $A \rightarrow \infty$ while $m A$ is kept constant. Moreover, the limit in a Minkowski background was obtained [5] using boost-rotation symmetric coordinates which are related to the Weyl form of the metric and this does not exist when the cosmological constant is non-zero. However, we can perform the transformation

$$
\left.\begin{array}{c}
t+\int \frac{\mathrm{d} y}{F}=A u \\
x+y=\frac{1}{A r} \\
\phi=-i \frac{\zeta-\bar{\zeta}}{\sqrt{2}} \\
\int \frac{\mathrm{d} x}{G}=A u-\frac{\zeta+\bar{\zeta}}{\sqrt{2}}
\end{array}\right\} \Leftrightarrow\left\{\begin{array}{c}
r=\frac{1}{A(x+y)} \\
u=\frac{1}{A}\left(t+\int \frac{\mathrm{d} y}{F}\right) \\
\zeta=\frac{1}{\sqrt{2}}\left(t+\int \frac{\mathrm{d} y}{F}-\int \frac{\mathrm{d} x}{G}+i \phi\right)
\end{array}\right.
$$

which puts the metric (17) into the form of the Robinson-Trautman solutions

$$
\mathrm{d} s^{2}=2 \mathrm{~d} u \mathrm{~d} r+2 H \mathrm{~d} u^{2}-2 \frac{r^{2}}{P^{2}} \mathrm{~d} \zeta \mathrm{d} \bar{\zeta}
$$

where

$$
2 H(r, u, \zeta, \bar{\zeta})=\Delta(\log P)-2 r(\log P)_{u}-\frac{2 m}{r}-\frac{\Lambda}{3} r^{2},
$$

with $\Delta=2 P^{2} \partial_{\zeta} \partial_{\bar{\zeta}}$, and with the special expression for $P$ given by

$$
P(\zeta, \bar{\zeta}, u)=G^{-1 / 2}(x(\zeta, \bar{\zeta}, u))
$$

It can be shown, as required, that (19) satisfies the Robinson-Trautman (vacuum) equation.

This form of the C-metric has the immediate advantage that the dependence on $A$ and $\Lambda$ is completely separated. The cosmological constant $\Lambda$ only occurs linearly in the expression for $H$, 
whereas the acceleration $A$ appears in the metric function $P$ through (19) in which $x(\zeta, \bar{\zeta}, u)$ has to be substituted using

$$
\int \frac{\mathrm{d} x}{G}=\int \frac{\mathrm{d} x}{1-x^{2}-2 m A x^{3}}=A u-\frac{1}{\sqrt{2}}(\zeta+\bar{\zeta}) .
$$

The cubic $G$ possess three distinct roots provided $0<27 m^{2} A^{2} \leq 1$ which are given by

$$
\begin{aligned}
& x_{1}=-\frac{1}{6 m A}\left[1+2 \cos \left(\varphi+\frac{2}{3} \pi\right)\right], \\
& x_{2}=-\frac{1}{6 m A}\left[1+2 \cos \left(\varphi+\frac{4}{3} \pi\right)\right], \\
& x_{3}=-\frac{1}{6 m A}[1+2 \cos \varphi],
\end{aligned}
$$

where

$$
\varphi=\frac{1}{3} \cos ^{-1}\left(1-54 m^{2} A^{2}\right)
$$

It can be seen that $x_{3}<x_{2}<0<x_{1}$ and, when $m A$ is small, $x_{1} \approx 1-m A, x_{2} \approx-1-m A$, and $x_{3} \approx-1 /(2 m A)$.

For the standard C-metric, $x$ must be in the range $x_{2} \leq x \leq x_{1}$, and we can express $G(x)$ in the form

$$
G(x)=2 m A\left(x_{1}-x\right)\left(x-x_{2}\right)\left(x-x_{3}\right) .
$$

Thus, we can write

$$
-\frac{1}{G(x)}=\frac{\alpha}{x-x_{1}}+\frac{\beta}{x-x_{2}}+\frac{\gamma}{x-x_{3}}
$$

where

$$
\begin{aligned}
& \alpha^{-1}=2 m A\left(x_{1}-x_{2}\right)\left(x_{1}-x_{3}\right), \\
& \beta^{-1}=-2 m A\left(x_{1}-x_{2}\right)\left(x_{2}-x_{3}\right), \\
& \gamma^{-1}=2 m A\left(x_{1}-x_{3}\right)\left(x_{2}-x_{3}\right),
\end{aligned}
$$

and $\alpha+\beta+\gamma=0$. Using (21), we have

$$
x_{1}-x_{2}=\frac{1}{\sqrt{3} m A} \sin \varphi, \quad x_{1}-x_{3}=\frac{1}{\sqrt{3} m A} \sin \left(\varphi+\frac{\pi}{3}\right), \quad x_{2}-x_{3}=\frac{1}{\sqrt{3} m A} \sin \left(\varphi+\frac{2 \pi}{3}\right),
$$

so that $\alpha, \beta$ and $\gamma$ can be expressed as

$$
\alpha=\frac{6 m A}{2 \cos \left(2 \varphi-\frac{2 \pi}{3}\right)+1}, \quad \beta=\frac{-6 m A}{2 \cos \left(2 \varphi-\frac{\pi}{3}\right)-1}, \quad \gamma=\frac{6 m A}{2 \cos (2 \varphi)+1},
$$

where $\varphi$ is given by (22). It can be seen that $\alpha$ and $\beta$ are monotonically decreasing functions of $m A$, while $\gamma$ is an increasing function and the ranges are given by

$$
\frac{1}{2} \geq \alpha>\frac{2}{3 \sqrt{3}}, \quad-\frac{1}{2} \geq \beta>-\infty, \quad 0 \leq \gamma<\infty .
$$

It is now possible to integrate (20) to obtain

$$
\left(x_{1}-x\right)^{\alpha}\left(x-x_{2}\right)^{\beta}\left(x-x_{3}\right)^{\gamma}=B e^{-A u} e^{(\zeta+\bar{\zeta}) / \sqrt{2}},
$$

where $B$ is a constant that will be determined later. This is an implicit equation for $x$ which should be substituted into the above expression (19) for $P$. In the limit as $A \rightarrow \infty$ while $m A$ is kept constant, we expect that the solution should approach that of an impulsive expanding wave in a Minkowski, de Sitter or anti-de Sitter background together with an associated snapping cosmic string. However, it can be seen that the above equation for $x$ becomes degenerate in this limit in which $x \rightarrow x_{1}$ for $u>0$ and $x \rightarrow x_{2}$ for $u<0$. Nevertheless, it is possible to overcome 
this problem using the permitted coordinate freedom $u \rightarrow U(u), \quad r \rightarrow r / \dot{U}, \quad P \rightarrow P / \dot{U}$, $m \rightarrow m / \dot{U}^{3}$.

Let us first consider the case for $U>0$. In the limit as $x \rightarrow x_{1}$, (23) implies that

$$
x_{1}-x \approx B^{1 / \alpha}\left(x_{1}-x_{2}\right)^{-\beta / \alpha}\left(x_{1}-x_{3}\right)^{-\gamma / \alpha} e^{-A U / \alpha} e^{(\zeta+\bar{\zeta}) / \sqrt{2} \alpha},
$$

so that

$$
P^{2} \approx C^{2} \dot{U}^{2} e^{A U / \alpha} e^{-(\zeta+\bar{\zeta}) / \sqrt{2} \alpha},
$$

where

$$
C^{2}=\frac{1}{2 m A} B^{-1 / \alpha}\left(x_{1}-x_{2}\right)^{(\beta-\alpha) / \alpha}\left(x_{1}-x_{3}\right)^{(\gamma-\alpha) / \alpha} .
$$

To ensure that the expression for $P$ remains finite as $A \rightarrow \infty$, we use our coordinate freedom to set $C \dot{U}=e^{-A U / 2 \alpha}$ for $U>0$. Having made this transformation, the limit $A \rightarrow \infty$ for $U>0$ leads to

$$
P^{2}=e^{-(\zeta+\bar{\zeta}) / \sqrt{2} \alpha}
$$

Similarly, for $U<0$, in the limit as $x \rightarrow x_{2}$ (23) implies that

$$
x-x_{2} \approx B^{1 / \beta}\left(x_{1}-x_{2}\right)^{-\alpha / \beta}\left(x_{2}-x_{3}\right)^{-\gamma / \beta} e^{-A U / \beta} e^{(\zeta+\bar{\zeta}) / \sqrt{2} \beta},
$$

and

$$
P^{2} \approx D^{2} \dot{U}^{2} e^{A U / \beta} e^{-(\zeta+\bar{\zeta}) / \sqrt{2} \beta},
$$

where

$$
D^{2}=\frac{1}{2 m A} B^{-1 / \beta}\left(x_{1}-x_{2}\right)^{(\alpha-\beta) / \beta}\left(x_{2}-x_{3}\right)^{(\gamma-\beta) / \beta} .
$$

To ensure that this remains finite as $A \rightarrow \infty$, we can again use our coordinate freedom to set $C \dot{U}=e^{-A U / 2 \beta}$ for $U>0$. Having made this transformation, the limit $A \rightarrow \infty$ for $U<0$ leads to

$$
P^{2}=\frac{D^{2}}{C^{2}} e^{-(\zeta+\bar{\zeta}) / \sqrt{2} \beta} .
$$

The function $U(u)$ as specified above satisfies the conditions that $U>0$ when $u>0$ and $U<0$ when $u<0$. It is given explicitly by

$$
U(u)=\left\{\begin{array}{lll}
\frac{2 \alpha}{A} \log \left(1+\frac{A}{2 \alpha C} u\right) & \text { for } & u>0 \\
\frac{2 \beta}{A} \log \left(1+\frac{A}{2 \beta C} u\right) & \text { for } & u<0
\end{array}\right.
$$

Including the additional value $U=0$ when $u=0$, the above transformation is $C^{1}$, and the resulting function $U(u)$ is monotonically increasing with $U<0$ for $u<0$ and $U>0$ for $u>0$ since $\alpha>0$ and $\beta<0$.

Before constructing the metric function $P^{2}(\zeta, \bar{\zeta}, u)$ explicitly for the whole range of $u$, however, we must observe that according to (23) the two roots $x=x_{1}$ and $x=x_{2}$ correspond to the coordinate limits $\zeta \rightarrow-\infty$ and $\zeta \rightarrow \infty$ respectively. Thus, the expressions (24) and (25) correspond to expansions about opposite poles of the spherical wave surfaces. In order to maintain a continuous coordinate system, it is necessary to make the transformation $\zeta \rightarrow-\zeta$ in one of the two regions. (The necessity of this procedure can be confirmed in the limit when $m=0$. See also figure 2) Choosing to perform this in the region $u>0$, we obtain

$$
P^{2}(\zeta, \bar{\zeta}, u)=\left\{\begin{array}{lll}
P_{+}^{2}=\exp \left(\frac{\zeta+\bar{\zeta}}{\sqrt{2} \alpha}\right) & \text { for } & u>0 \\
P_{-}^{2}=\frac{D^{2}}{C^{2}} \exp \left(\frac{\zeta+\bar{\zeta}}{-\sqrt{2} \beta}\right) & \text { for } & u<0
\end{array}\right.
$$



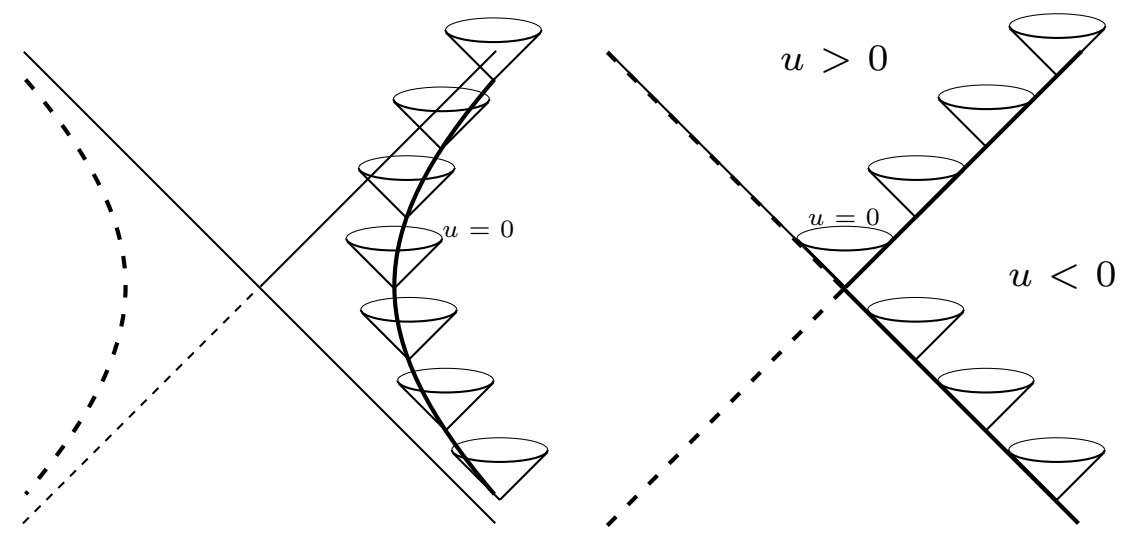

Figure 2: In Robinson-Trautman coordinates, the null surfaces $u=$ const. define a family of null cones centred on one accelerating black hole. These foliate half of the complete space-time (above the diagonal in the left diagram) which is symmetric about the vertical axis. In the limit as $A \rightarrow \infty$, the "source" of these cones approaches the null asymptotes as in the diagram on the right. The null surfaces still foliate half of the space-time, but are stacked in different ways in the two halves $u<0$ and $u>0$. There is an obvious discontinuity in the foliation across the impulsive wave at $u=0$. These schematic pictures apply in Minkowski, de Sitter and anti-de Sitter backgrounds.

Although the discontinuity at $u=0$ is consistent with the presence of an impulsive wave, this is not yet expressed in the form given above for an impulsive Robinson-Trautman solution. However, we can now make use of the remaining coordinate freedom that $\zeta \rightarrow \tilde{\zeta}=h(\zeta)$ under which $P^{2} \rightarrow \tilde{P}^{2}=h^{\prime} \bar{h}^{\prime} P^{2}$. In particular, we can make the specific choice $h=\sqrt{2} \alpha \exp (-\zeta / \sqrt{2} \alpha)$ so that

$$
\tilde{P}_{+}^{2}=1, \quad \tilde{P}_{-}^{2}=\frac{D^{2}}{C^{2}}\left(\frac{\tilde{\zeta} \tilde{\tilde{\zeta}}}{2 \alpha^{2}}\right)^{1+(\alpha / \beta)} .
$$

Dropping the tildes, we can then put $g=-\alpha / \beta \in(0,1]$ and choose the constant $B$ such that $2 \beta^{2}\left(2 \alpha^{2}\right)^{\alpha / \beta} C^{2}=D^{2}$, i.e. $B^{(1 / \alpha)-(1 / \beta)}=\left(2 \alpha^{2}\right)^{1+\alpha / \beta}(\alpha / \gamma)^{\alpha / \beta}(-\gamma / \beta)^{\beta / \alpha}$. We thus finally obtain

$$
P^{2}(\zeta, \bar{\zeta}, u)=\left\{\begin{array}{ccc}
1 & \text { for } & u>0 \\
g^{-2}(\zeta \bar{\zeta})^{1-g} & \text { for } & u<0
\end{array}\right.
$$

which is exactly the form given above for an impulsive Robinson-Trautman wave (13) with $\epsilon=0$ and $g$ given by (15). In fact, it can be seen from figure 2 that the foliation of the space-time in either regions $u<0$ or $u>0$ by the null cones $u=$ const. is only consistent with the parametrization of the Robinson-Trautman family of solutions with $\epsilon=0$ as described in [16]. In this parametrization, the solution (27) describes an impulsive spherical wave generated by the snapping of a cosmic string with deficit angle $2 \pi(1-g)$ in a Minkowski, de Sitter or anti-de Sitter background according to the value of $\Lambda$. It reduces to the corresponding complete background with no string and no impulse when $m A=0$, in which case $\alpha=\frac{1}{2}=-\beta, g=1$, and $B=1$ so that $P=1$ everywhere. It also agrees with the null limit of the C-metric [5] that was previously obtained only for the Minkowski background.

\section{Concluding remarks}

The form of the C-metric considered above describes a pair of black holes which accelerate away from each other in a Minkowski, de Sitter or anti-de Sitter universe under the action of cosmic strings which pull them apart. By adopting a Robinson-Trautman coordinate system, we have shown that this type D space-time leads to a solution which describes a snapping cosmic string 
in any of these backgrounds in the limit in which the acceleration $A$ becomes unbounded while the mass $m$ is scaled to zero such that $m A$ is kept constant. This procedure leads to a metric form which is discontinuous over the spherical wavefront $u=0$ generated by the snapping string. Such a discontinuity seems to be inevitable in this coordinate system due to the difference in the foliation of the space-time in the two regions $u<0$ and $u>0$ as illustrated in figure 2 . Nevertheless, the interpretation given above is justified because the limit obtained is identical to that which arises as the impulsive limit of a specific family of type $\mathrm{N}$ sandwich waves of Robinson-Trautman type.

In section 4, we considered such a special family of Robinson-Trautman type $\mathrm{N}$ spacetimes which describe expanding sandwich waves with spherical wavefronts again in Minkowski, de Sitter or anti-de Sitter backgrounds [16]. These were given for all possible values of the parameter $\epsilon$ which determines the foliation of the space-time. The family of solutions considered were previously shown to contain a pair of cosmic strings ahead of the wave, but have no string behind it. Apart from the presence of a string-like singularity within the wave region, these solutions are regular and well behaved everywhere. It is then very reasonable to investigate these solutions in the limit as the duration of the wave becomes arbitrarily small. Such a limit leads unambiguously to an expression for a spherical impulsive gravitational wave, but the metric again becomes discontinuous and is exactly the null limit of the C-metric described above.

In section 3, we used the "cut and paste" method as another way to construct spherical impulsive waves. Here, we presented a solution in which the metric is explicitly continuous and also valid for all possible values of the cosmological constant and the parameter $\epsilon$. These provide an alternative form of the above solutions for a snapping string together with an expanding impulse. We have indicated how this form of the metric is related to the 5-dimensional representation of the background de Sitter and anti-de Sitter universes.

When $\Lambda=0$, the null limit of the C-metric was previously derived in an explicitly continuous form [5]. This could be achieved because a global coordinate system adapted to the boostrotation symmetry was available. However, such coordinates are still unknown for the C-metric with a non-zero cosmological constant. Nevertheless, we have been able to derive the solution for a snapping cosmic string generating an expanding impulsive spherical gravitational wave in a de Sitter and anti-de Sitter universe by three alternative methods. These different approaches complement each other and lead to a fairly complete understanding of these space-times.

\section{Acknowledgements}

The work of JP was partly supported by the grants GAČR-202/02/0735, GAUK 166/2003 and the London Mathematical Society.

\section{References}

[1] Gleiser R and Pullin J 1989 Class. Quantum Grav. 6 L141

[2] Bičák J 1990 Astron. Nachr. 311189

[3] Bičák J and Schmidt B 1989 Class. Quantum Grav. 61547

[4] Podolský J and Griffiths J B 2001 Gen. Rel. Grav 3337

[5] Podolský J and Griffiths J B 2001 Gen. Rel. Grav 3359

[6] Penrose R 1972 General Relativity ed L O'Raifeartaigh (Oxford: Clarendon)

[7] Nutku Y and Penrose R 1992 Twistor Newsletter No. 34, 11 May, 9

[8] Hogan P A 1993 Phys. Rev. Lett. 70117 
[9] Hogan P A 1994 Phys. Rev. D 496521

[10] Aliev A N and Nutku Y 2001 Class. Quantum Grav. 18891

[11] Hogan P A 1992 Phys. Lett. A 17121

[12] Podolský J and Griffiths J B 2000 Class. Quantum Grav. 171401

[13] Podolský J and Griffiths J B 1999 Class. Quantum Grav. 162937

[14] García Díaz A and Plebański J F 1981 J. Math. Phys. 222655

[15] Griffiths J B and Docherty P 2002 Class. Quantum Grav. 19 L109

[16] Griffiths J B, Podolský J and Docherty P 2002 Class. Quantum Grav. 194649

[17] Salazar H, García Díaz A and Plebański J F 1983 J. Math. Phys. 242191

[18] Plenański J F and Demianski M 1976 Ann. Physics 9898

[19] Podolský J and Griffiths J B 2001 Phys. Rev. D 63, 024006

[20] Dias O J C and Lemos J P S 2003 Phys. Rev. D 67, 064001

[21] Dias O J C and Lemos J P S 2003 Phys. Rev. D 67, 084018

[22] Krtouš P and Podolský J 2003 Phys. Rev. D 68, 024005

[23] Podolský J, Ortaggio M and Krtouš P 2003 Phys. Rev. D 68, 124004 